Open Pharmaceutical Sciences Journal
CrossMark
Content list available at: www.benthamopen.com/PHARMSCI
DOI: $10.2174 / 1874844901603010117$

RESEARCH ARTICLE

\title{
Microwave Assisted Facile Synthesis and Biological Evaluation of Novel 2-Indolyl -1, 5-Benzothiazepines
}

\author{
Anna P. G. Nikalje ${ }^{*}$, , Mangesh S. Ghodke ${ }^{b}$, Firoz A. K. Khan ${ }^{a}$ and Jaiprakash N. Sangshetti ${ }^{a}$ \\ ${ }^{a}$ Department of Pharmaceutical Chemistry, Y.B. Chavan College of Pharmacy, Dr. Rafiq Zakaria Campus, Aurangabad \\ 431003 (M.S.) India \\ ${ }^{b}$ Department of Pharmaceutical Chemistry, R. C. Patel Institute of Pharmaceutical Education \& Research, \\ Shirpur-425405, Dhule (M.S.) India
}

Received: June 11, 2015

Revised: March 21, 2016

Accepted: March 25, 2016

\begin{abstract}
:
Background:

The research work reports facile, eco-friendly microwave- assisted solvent free synthesis of coupled heterocyclic system 2-(1Hindol-3-yl)-4-substitued-2, 3-dihydrobenzo [1,5] thiazepine derivatives obtained by cyclo condensation of 1 -substituted-3(1Hindolyl)-2-propen-1-ones with 2-amino thiophenol in presence of eco-friendly catalyst zirconium(IV) oxy chloride, in solvent-free conditions. The reaction was completed in 3-6 minutes and gives better yields than the conventional synthesis which requires 6-8 hrs.

\section{Result and Conclusion:}

The newly synthesized compounds were evaluated for antihypertensive activity in Sprague- Dawley rats by tail- cuff method and compared with diltiazem, the standard antihypertensive drug. The data suggested that some of the compounds of the current series exhibited enhanced antihypertensive activity than the standard. As benzothiazepines are bioisosters of benzodiazepines, the synthesized novel indolyl-benzothiazepine derivatives were also screened for CNS activities such as CNS depressant activity by actophotometer and anticonvulsant activity by MES and PTZ model on mice. The title compounds have exhibited good CNS depression and anticonvulsant activity. The compounds thus have shown dual antihypertensive and CNS depressant, anticonvulsant activity and are biologically potential molecules. The molecular docking was performed for the synthesized compounds to assess their binding affinities to GABA-A receptor in order to rationalize their anticonvulsant activities in a qualitative way.
\end{abstract}

Keywords: 1, 5-benzothiazepine, Antihypertensive activity, CNS depressant, MES, Microwave-assisted, PTZ.

\section{INTRODUCTION}

Over the last several decades, tremendous advances in basic and clinical research on cardiovascular disease have greatly improved the prevention and treatment of the cardiovascular diseases, the nation's number one killer of men and women of all races $[1,2]$. Hypertension is a multi factorial disease frequently associated with other cardiovascular problems. None of the antihypertensive drugs available can cure all cases of hypertension. This situation causes a unique scenario in this area of therapeutic research: less recent approaches, which make use of drugs acting on the adrenergic system, diuretics, calcium antagonists, nitro-vasodilators and so on, have not yet been abandoned, while new compounds are still being developed today. Excessive vasodilatation results in hypotension, dizziness and headache Dihydropyridines are often associated with tachycardia and chest pain, whereas verapamil and diltiazem may cause bradycardia, also associated with additive cardio depressive effects. Keeping inview the present stressful lifestyle,

\footnotetext{
* Address correspondence to this author at the Department of Pharmaceutical Chemistry, Y.B. Chavan College of Pharmacy, Dr. Rafiq Zakaria Campus, Rauza Bagh, P.B. No. 33, Aurangabad, Maharashtra 431001, India; Fax: +91 0240 2381129; E-mail: annapratimanikalje@gmail.com
} 
eating habits, insufficient regular physical exercise, smoking and obesity most people are likely to suffer from disorders related to heart, such as congestive heart failure, angina, hypertension, arrhythmias etc, that necessitates study and search for more effective drug molecules.

The drug 'diltiazem' being used as a calcium channel blocker and modulator [3, 4], calcium channel antagonist [5], vasodilator [6], antihypertensive [7, 8], blood platelet aggregation inhibitor [9], antiarrhythmic [10], antiischemic [11], etc., possesses a 1,5-benzothiazepine nucleus having a p-methoxy phenyl group at position-2, a 4-oxo group with no substituent in the fused benzene ring. Beta blockers such as carvedilol contains indole, therefore, we thought it worthwhile to synthesize a new hybrid heterocyclic moiety containing 1, 5-benzothiazepine and indole ring coupled together with the hope to get enhanced antihypertensive activity. In addition, benzothiazepines are the versatile pharmacophores having various biological activities such as antimicrobial activity [12] CNS depressant, anticonvulsant activity [13, 14]. Valium or Dizepam, is 7-chloro-1-methyl-5- phenyl-3H-1, 4-benzodiazepin-2-one, a drug used as an anti anxiety agent, sedative, antipanic agent, antitremor agent and skeletal muscle relaxant. Benzothiazepine is an example of bioisosteric replacement of benzodiazepines, i.e., benzothiazepines has structural resemblance with benzodiazepines. Indole is the most important heterocyclic nucleus which has gained prominence in medicinal chemistry due to its diverse biological activities such as anticonvulsant [15 - 20] anti-inflammatory [21, 22], and antipsychotic [23] activities. A Hybrid drug which comprises the incorporation of two drug pharmacophores in one single molecule are basically designed to interact with multiple targets or to amplify its effect through action on another bio target as one single molecule or to counterbalance the known side effects associated with the other hybrid part. Due to the potential biological activities associated with these two moieties and in continuation of our earlier research work on 1,5-benzothizepines and indole [24, 25] it was thought worthwhile to synthesize and evaluate the novel benzothiazepine-indole derivatives in a single molecular frame work as shown in Fig. (1), for antihypertensive and CNS activities as well, with the hope to get better CNS depressant and anticonvulsant agents with lower toxicity. Molecular docking studies have also been performed for anticonvulsant activity of synthesized compounds with gamma aminobutryic acid-A (GABA-A) in order to identify possible binding interactions with the ligand. The present investigation is the report of facile, eco-friendly microwave- assisted cyclo condensation of 1 -substituted-3(1Hindolyl)-2-propen-1-ones with 2-amino thiophenol in presence of catalyst zirconium oxy chloride, in solvent-free conditions under microwave irradiation to afford the coupled heterocyclic system 2-(1H-indol-3-yl)-4-substitued-2, 3dihydrobenzo [1,5] thiazepine derivatives and its biological evaluation as antihypertensive and CNS depressant anticonvulsant agents.

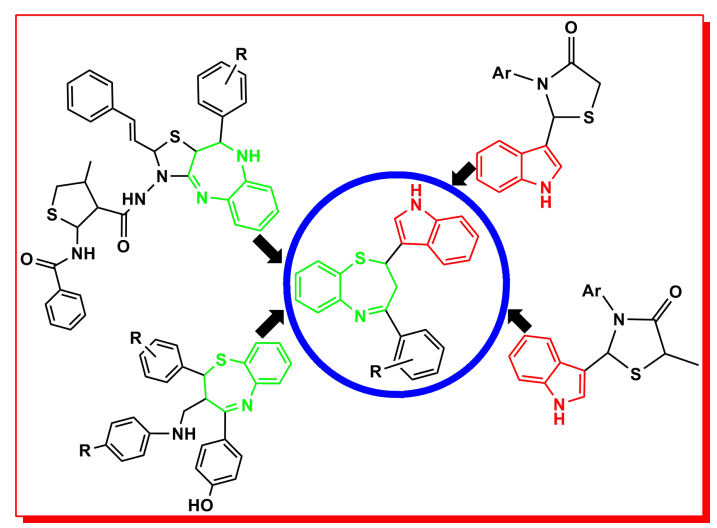

Fig. (1). Designing protocol.

\section{RESULTS AND DISCUSSION}

\section{Chemistry}

In recent years, one of the most straight forward protocols used for the syntheses of 1, 5-benzothiazepines has been the cyclo condensation reaction of $\alpha, \beta$-unsaturated carbonyl compounds, such as chalcones with 2-aminothiophenols. Literature survey reports such reactions [26, 27] under various reaction conditions by using i) acidic mediummethanol/ethanol containing glacial acetic acid; ethanol saturated with hydrogen chloride gas; toluene containing traces of trifluoro acetic acid, ii) basic medium-pyridine or toluene containing piperidine and iii) neutral medium- anhydrous toluene or o-xylene. However, the combination of solvents having strong acid and long reaction time period makes 
these methods environmentally hazardous. Therefore, a simple, general and efficient procedure for the synthesis of this important heterocyclic system is required. The application of microwave irradiation to organic synthesis has been focus of considerable attention in recent years and is becoming an increasingly popular technology [28]. In the present work, we have synthesized the coupled molecules containing indole and 1, 5-benzothiazepine nucleus where indole was used as starting material and benzothiazepine ring was developed on the indole ring, in solvent free conditions in presence of eco-friendly catalyst zirconium (IV) oxy chloride. Zirconium (IV) oxy chloride is an efficient Lewis catalyst used in various transformations, such as electrophilic addition reaction of indole with aldehydes /ketones to afford bis indolyl methanes in good yield [29]. However, there are no reports of its use as a catalyst for synthesis of indolyl nucleus coupled with 1, 5-benzothiazepine nucleus. This eco-friendly catalyst is cheaper in cost and is water-soluble, hence it is easy to remove off from the reaction mixture. In this regard, it was planned to introduce formyl group in the indole ring to form indole-3-carbaldehyde, and condense with various aromatic ketones to form chalcones or 1-substituted-3(1Hindolyl)-2-propen-1-ones, and their cyclo condensation with 2-aminothiophenol to give 1, 5-benzothiazepine bearing indole moiety at $\mathrm{C} 2$ position and various aromatic substituent's at C4. Final compounds 2-(1H-indol-3-yl)-4substituted-2,3-dihydrobenzo- [1,5] thiazepine 4(a-o) were synthesized as outlined in Scheme 1 under microwave irradiation in solvent free condition, following green chemistry principles in good yields and enhanced reaction rates. Commercially available starting material indole 1 was reacted with phosphorus oxychloride and dimethylformamide to form indole-3-carbaldehyde. Formylation of $\mathbf{1}$ according to a procedure reported by Vilsmeier-Haack afforded indole-3carbaldehyde 2. Further, Claisen- Schmidt condensation of $\mathbf{2}$ with various ketones and substituted acetophenones in the presence of ethanol and sodium hydroxide forms chalcones or 1-substituted-3(1H-indolyl)-2-propen-1-ones 3(a-o). Cyclo condensation of chalcones 3(a-o) with 2-aminothiophenol in the presence of catalytic amount of zirconyl oxy chloride afforded final compounds, i.e., indolyl -1, 5-benzothiazepine derivatives 4(a-o). The reaction was completed in 3-6 minutes at power selector level 700 Watts at temperature $80^{\circ} \mathrm{C}$, which was recorded by temperature probe of the microwave. The microwave-irradiated solvent-free synthesis gives better yields in shorter time than the conventional synthesis which requires 6-8 hrs refluxing in solvent. The structures of the final compounds $\mathbf{4 ( a - o )}$ were assigned on the basis of spectral analysis. The spectral data is given in Supplementary File. Characterization data of the synthesized derivatives is given in Table 1 . Most of the derivatives were obtained in $65-82 \%$ yield by microwave technique within 3-5 min of reaction. Synthesized compounds were confirmed by FTIR, Mass, ${ }^{1}$ HNMR spectroscopic analysis. All the spectral data are in accordance with assumed structures. IR spectra were scanned on FTIR-PS 4000, within 4000-400 $\mathrm{cm}^{-1}$ wavelength range. $\mathrm{KBr}$ power technique was used for sampling purpose. In the IR spectra of indole-3-carbaldehyde $-\mathrm{NH}$ stretching occurs at $3448 \mathrm{~cm}^{-1}$, -CHO stretching $2710 \mathrm{~cm}^{-1}$; in case of NMR spectra multiplates observed at $7.2-8.1$ $\delta \mathrm{ppm}$ for aromatic protons, singlet obeserved at $9.7 \delta \mathrm{ppm}$ for $\mathrm{CHO}$ and singlet at $10.8 \delta \mathrm{ppm}$ for $-\mathrm{NH}$ proton of indole, mass peak observed at 145 which confirms the formation of indole-3-carbaldehyde.

Table 1. Physical characterization of final indolyl -1, 5- benzothiazepines 4(a-o).

\begin{tabular}{|c|c|c|c|c|c|}
\hline Compounds & $\mathbf{R}$ & Time (min) & $\begin{array}{c}\text { Yield (\%) } \\
\text { (Microwave) }\end{array}$ & mp (0C) & Molecular formula \\
\hline $4 \mathrm{a}$ & $\mathrm{CH}_{3}$ & 3.5 & 75 & 80 & $\mathrm{C}_{18} \mathrm{H}_{16} \mathrm{~N}_{2 \mathrm{M}} \mathrm{S}$ \\
\hline $4 \mathrm{~b}$ & $\mathrm{C}_{2} \mathrm{H}_{5}$ & 3 & 82 & 100 & $\mathrm{C}_{19} \mathrm{H}_{18} \mathrm{~N}_{2} \mathrm{~S}$ \\
\hline $4 \mathrm{c}$ & $\mathrm{C}_{6} \mathrm{H}_{5}$ & 5 & 65 & 110 & $\mathrm{C}_{23} \mathrm{H}_{18} \mathrm{~N}_{2} \mathrm{~S}$ \\
\hline $4 \mathrm{~d}$ & $4-\mathrm{ClC}_{6} \mathrm{H}_{4}$ & 4 & 65 & 90 & $\mathrm{C}_{23} \mathrm{H}_{17} \mathrm{~N}_{2} \mathrm{C}_{1} \mathrm{~S}$ \\
\hline $4 \mathrm{e}$ & $4-\mathrm{FC}_{6} \mathrm{H}_{4}$ & 3.5 & 74 & 65 & $\mathrm{C}_{23} \mathrm{H}_{17} \mathrm{~N}_{2} \mathrm{FS}$ \\
\hline $4 \mathrm{f}$ & $4-\mathrm{NO}_{2} \mathrm{C}_{6} \mathrm{H}_{4}$ & 6 & 65 & 125 & $\mathrm{C}_{23} \mathrm{H}_{17} \mathrm{O}_{2} \mathrm{~N}_{3} \mathrm{~S}$ \\
\hline $4 \mathrm{~g}$ & $4-\mathrm{OHC}_{6} \mathrm{H}_{4}$ & 5 & 70 & 120 & $\mathrm{C}_{23} \mathrm{H}_{18} \mathrm{ON}_{2} \mathrm{~S}$ \\
\hline $4 \mathrm{~h}$ & $4-\mathrm{OCH}_{3} \mathrm{C}_{6} \mathrm{H}_{4}$ & 4 & 75 & 90 & $\mathrm{C}_{24} \mathrm{H}_{20} \mathrm{ON}_{2} \mathrm{~S}$ \\
\hline $4 \mathrm{i}$ & $2-\mathrm{OHC}_{6} \mathrm{H}_{4}$ & 5 & 70 & 130 & $\mathrm{C}_{23} \mathrm{H}_{18} \mathrm{ON}_{2} \mathrm{~S}$ \\
\hline $4 \mathrm{j}$ & $4-\mathrm{OCH}_{2} \mathrm{COOHC}_{6} \mathrm{H}_{4}$ & 4.5 & 45 & 103 & $\mathrm{C}_{25} \mathrm{H}_{20} \mathrm{O}_{3} \mathrm{~N}_{2} \mathrm{~S}$ \\
\hline $4 \mathrm{k}$ & $4-\mathrm{NH}_{2} \mathrm{C}_{6} \mathrm{H}_{4}$ & 4 & 75 & 80 & $\mathrm{C}_{23} \mathrm{H}_{19} \mathrm{~N}_{2} \mathrm{~S}$ \\
\hline 41 & $3-\mathrm{OCH}_{3} \mathrm{C}_{6} \mathrm{H}_{4}$ & 4.5 & 80 & 110 & $\mathrm{C}_{24} \mathrm{H}_{20} \mathrm{~N}_{2} \mathrm{OS}$ \\
\hline $4 \mathrm{~m}$ & $3-\mathrm{OHC}_{6} \mathrm{H}_{4}$ & 5 & 65 & 125 & $\mathrm{C}_{23} \mathrm{H}_{18} \mathrm{~N}_{2} \mathrm{OS}$ \\
\hline $4 \mathrm{n}$ & $3,4-\mathrm{OCH}_{3} \mathrm{C}_{6} \mathrm{H}_{3}$ & 4 & 75 & 105 & $\mathrm{C}_{25} \mathrm{H}_{22} \mathrm{~N}_{2} \mathrm{O}_{2} \mathrm{~S}$ \\
\hline $4 \mathrm{o}$ & $2-\mathrm{NO}_{2} \mathrm{C}_{6} \mathrm{H}_{4}$ & 5 & 80 & 135 & $\mathrm{C}_{23} \mathrm{H}_{17} \mathrm{~N}_{3} \mathrm{O}_{2} \mathrm{~S}$ \\
\hline
\end{tabular}

For the derivative i.e. 2-(1H-indol-3-yl)-4-ethyl-2, 3-dihydro-1, 5-benzothiazepine 4a, in IR spectra -NH stretching occurs at $3438 \mathrm{~cm}^{-1},-\mathrm{C}=\mathrm{N}$ stretching at $1590 \mathrm{~cm}^{-1}$, for NMR spectra singlet for methyl group observed as singlet at 1.1 
$\delta \mathrm{ppm}$, -indole $-\mathrm{NH}$ peak observed at $10.1 \delta \mathrm{ppm}$. The formation of 1,5-benzothiazepines are confirmed by peculiar occurance of ${ }^{1} \mathrm{H}$ NMR double doublets in the region 1.8-2.2 $\delta$ ppm due to methylene protons at $\mathrm{C} 3$ position and 3.2-3.8 $\delta$ ppm due to methine protons of $\mathrm{C}-\mathrm{H}$ at $\mathrm{C} 2$ position. ${ }^{13} \mathrm{C} \operatorname{NMR}\left(\mathrm{CDCl}_{3}\right) \quad \delta$ ppm: 24.8, 41.3, 49.9,11.1,117.0,118.8,119.9,121.7,123.0, 125.8, 127.4, 133.4, 136.5, 137.6 and 164.6. Mass $(\mathrm{m} / \mathrm{z}): 292.40, \mathrm{M}+1$ peak at 293.10, M+2 peak at 294.10.The spectral data confirms the presence of two heterocyclic moities, i.e. indole and 1,5benzothiazepines in the final synthesized derivatives.

\section{Antihypertensive Activity}

All the synthesized compounds were evaluated for antihypertensive activity by in vivo mean blood pressure method in Sprague- Dawley rats according to a previously reported protocol using tail cuff apparatus. The blood pressure of each animal was then recorded before and after treatment with standard drug and test compounds using tail cuff pulse detector ML125/R which is connected to Power Lab Data Acquisition System. The data was recorded using Lab Chart 6 software and compared with that of the reference standard diltiazem $(5 \mathrm{mg} / \mathrm{kg}$ body weight). Data are expressed as Mean Blood Pressure \pm SEM. All the statistical calculations were carried out using Graph Pad ${ }^{\circledR}$ Prism 3.0 (USA) Statistical Software. Data was analyzed by one way ANOVA followed by Dunnett's test. Results were expressed as the mean \pm S.E. In vitro assay could not be done as the compounds were water- insoluble. The results for anti-hypertensive activity are given in Table $\mathbf{2}$. The data indicate that the compounds exhibited enhanced activity which can be attributed to the presence of indole moiety at position $\mathrm{C}_{2}$ and also due to the presence of electron donating groups at $\mathrm{C}_{4}$ such as methyl, phenyl and substituted phenyl groups.

Table 2. Mean systolic blood pressure before and after administration of the test compounds.

\begin{tabular}{|c|c|c|c|c|c|}
\hline \multirow{2}{*}{ Group } & \multicolumn{5}{|c|}{ Mean Blood Pressure \pm SEM } \\
\cline { 2 - 6 } & $\mathbf{0 ~ m i n .}$ & $\mathbf{1 5}$ min. & $\mathbf{3 0 ~ m i n .}$ & $\mathbf{6 0}$ min. & $\mathbf{9 0 ~ m i n . ~}$ \\
\hline Standard & $4.62 \pm 0.87$ & $1.98 \pm 2.15$ & $5.21 \pm 1.12$ & $12.49 \pm 0.30$ & $33.66 \pm 1.18$ \\
\hline $4 \mathrm{a}$ & $-0.77 \pm 3.71$ & $12.22 \pm 8.58$ & $18.82 \pm 8.74$ & $19.04 \pm 5.01$ & $8.34 \pm 11.08$ \\
\hline $4 \mathrm{~b}$ & $1.89 \pm 1.52$ & $7.95 \pm 1.95$ & $-1.16 \pm 1.67$ & $1.06 \pm 1.89$ & $2.84 \pm 2.50$ \\
\hline $4 \mathrm{c}$ & $-1.04 \pm 0.213$ & $3.01 \pm 2.6$ & $3.37 \pm 2.47$ & $21.80 \pm 1.28$ & $26.10 \pm 1.69$ \\
\hline $4 \mathrm{~d}$ & $-0.50 \pm 1.26$ & $-2.86 \pm 2.93$ & $-0.91 \pm 5.50$ & $-1.37 \pm 3.343$ & $1.46 \pm 4.04$ \\
\hline $4 \mathrm{e}$ & $-0.58 \pm 0.61$ & $16.07 \pm 0.05$ & $14.20 \pm 1.43$ & $1.10 \pm 1.28$ & $4.61 \pm 1.06$ \\
\hline $4 \mathrm{f}$ & $-4.34 \pm 5.59$ & $-2.01 \pm 5.01$ & $-8.04 \pm 8.74$ & $-31.20 \pm 14.77$ & $-31.77 \pm 12.93$ \\
\hline $4 \mathrm{~g}$ & $1.86 \pm 0.80$ & $18.06 \pm 1.69$ & $26.71 \pm 1.17$ & $28.03 \pm 0.94$ & $32.17 \pm 0.45$ \\
\hline $4 \mathrm{~h}$ & $0.78 \pm 0.60$ & $2.57 \pm 1.13$ & $37.55 \pm 1.89$ & $47.31 \pm 1.51$ & $49.97 \pm 2.05$ \\
\hline $4 \mathrm{i}$ & $0.24 \pm 0.59$ & $5.75 \pm 1.01$ & $21.23 \pm 1.02$ & $37.68 \pm 3.29$ & $27.30 \pm 1.45$ \\
\hline $4 \mathrm{j}$ & $-1.18 \pm 5.21$ & $9.69 \pm 4.42$ & $13.56 \pm 5.03$ & $12.51 \pm 6.94$ & $9.36 \pm 9.16$ \\
\hline
\end{tabular}

4h showed significant activity at 30,60 and $90 \mathrm{~min} .(P<0.001)$

$\mathbf{4 g}$ showed significant activity at 15,30 and $60 \min (P<0.05, P<0.01)$

$4 \mathbf{i}$ showed significant activity at 30 and $60 \mathrm{~min}(P<0.01)$

4a showed significant activity at 15 and $30 \mathrm{~min}(P<0.01)$

4e showed significant activity at 15 and $30 \min (P<0.01)$

Data was analyzed by one way ANOVA followed by Dunnett's test.

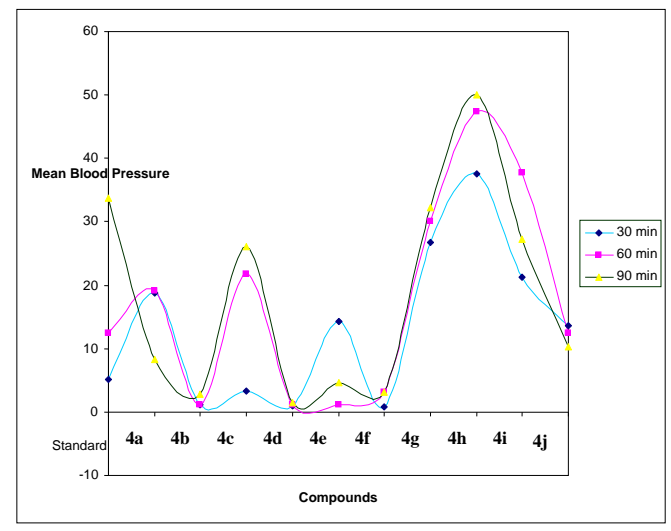

Fig. (2). Mean blood pressure vs drug treatment after 30, 60 and 90 min. 
Blood pressure was measured before and 15, 30, 60 and 90 min after drug administration, graphical result shown in Fig. (2). Diltiazem was administered as reference compound. Mean values in systolic blood pressure before and 15, 30, 60 and 90 min after drug administration were determined [30]. (CPCSEA Reg. No. CPN/ IAEC /08 /2008-09)

In comparison of the activities of compounds 4 (a-j) $(5 \mathrm{mg} / \mathrm{kg})$, compound $\mathbf{4 a}\left(-\mathrm{CH}_{3}\right)$ has activity similar to the reference standard drug diltiazem. However, compound $\mathbf{4 b}\left(-\mathrm{C}_{2} \mathrm{H}_{5}\right)$ did not reduce the blood pressure significantly, indicating that $-\mathrm{CH}_{3}$ group is optimum for the antihypertensive activity. Compound $\mathbf{4 c}$ where Ar is un substituted phenyl showed the antihypertensive activity i.e. decrease in blood pressure similar to that of reference standard drug diltiazem.

Among the synthesized derivatives, compound 4d (para-chloro phenyl ), 4e ( para-fluoro phenyl ) and to $4 \mathbf{f}$ (paranitro phenyl) with electron- withdrawing groups did not reduce the blood pressure significantly, demonstrating that the presence of electron withdrawing groups on the phenyl ring at position $\mathrm{C}_{4}$ decreases the antihypertensive activity.

In compounds $\mathbf{4 g}$ (p-hydroxy phenyl), $\mathbf{4 h}$ (para-methoxy phenyl) and $\mathbf{4 i}$ (ortho-hydroxy phenyl), reduced the blood pressure significantly. Thus, it was observed that electron- donating, polar groups at ortho or para position of phenyl ring increases the antihypertensive activity significantly, where as electron withdrawing groups on phenyl ring showed the reduced antihypertensive activity.

\section{CNS Depressant Activity}

The activity was measured as digital score using actophotometer [31] with the ip administration of drug (30 mg/kg) to mice.

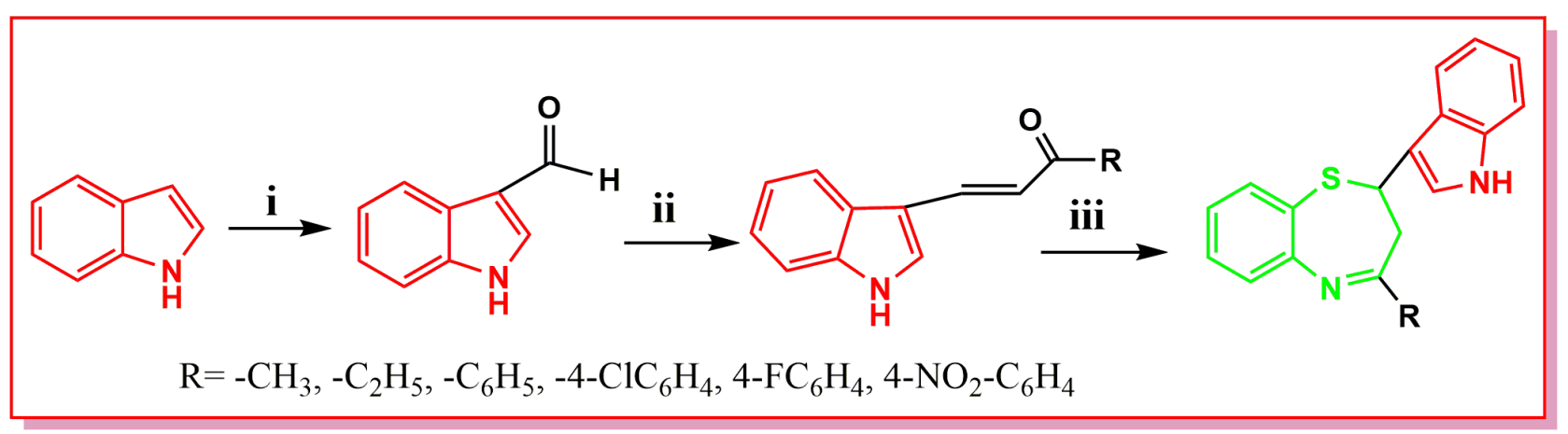

Scheme 1. Reagents and conditions (i) dimethylformamide, phosphoryl chloride, $\mathrm{NaOH}$, water, stirring (ii) ArCOCH3, ethanol, 40\% $\mathrm{NaOH}$, stir and kept for $24 \mathrm{hrs}$ at room temperature (iii) 2-aminothiophenol, zirconyl chloride, microwave irradiation.

4a)<smiles>CC1=Nc2ccccc2SC(c2c[nH]c3ccccc23)C1</smiles>

4b)<smiles>CCC1=Nc2ccccc2SC(c2c[nH]c3ccccc23)C1</smiles> 
4c)<smiles>c1ccc(C2=Nc3ccccc3SC(c3c[nH]c4ccccc34)C2)cc1</smiles>

4e)<smiles>Fc1ccc(C2=Nc3ccccc3SC(c3c[nH]c4ccccc34)C2)cc1</smiles>

4g)

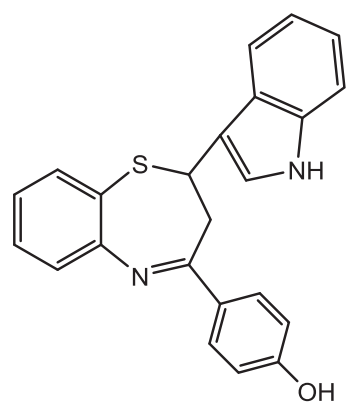

4i)<smiles>Oc1ccccc1C1=Nc2ccccc2SC(c2c[nH]c3ccccc23)C1</smiles>

4d)<smiles>Clc1ccc(C2=Nc3ccccc3SC(c3c[nH]c4ccccc34)C2)cc1</smiles>

4f)<smiles>O=[N+]([O-])c1ccc(C2=Nc3ccccc3SC(c3c[nH]c4ccccc34)C2)cc1</smiles>

4h)<smiles>COc1ccc(C2=Nc3ccccc3SC(c3c[nH]c4ccccc34)C2)cc1</smiles>

4j)<smiles>O=C(O)COc1ccc(C2=Nc3ccccc3SC(c3c[nH]c4ccccc34)C2)cc1</smiles> 
4k)<smiles>Nc1ccc(C2=Nc3ccccc3SC(c3c[nH]c4ccccc34)C2)cc1</smiles>

4m)<smiles>Oc1cccc(C2=Nc3ccccc3SC(c3c[nH]c4ccccc34)C2)c1</smiles>

41)<smiles>COc1cccc(C2=Nc3ccccc3SC(c3c[nH]c4ccccc34)C2)c1</smiles>

4n)<smiles>COc1ccc(C2=Nc3ccccc3SC(c3c[nH]c4ccccc34)C2)cc1OC</smiles>

4o)<smiles>O=[N+]([O-])c1ccccc1C1=Nc2ccccc2SC(c2c[nH]c3ccccc23)C1</smiles>

In behavioral activity using actophotometer, the compounds $\mathbf{4 c}$, $\mathbf{4 e}$ having $30 \mathrm{mg}$ dose have shown good CNS depressant activity when compared with standard drug diazepam. Test compounds with $100 \mathrm{mg}$ dose, 4a, 4e, 4f and 4g have shown good CNS depressant activity when compared with standard (diazepam, $4 \mathrm{mg} / \mathrm{kg}$ ). While compounds with $300 \mathrm{mg}$ dose, $\mathbf{4 a}, \mathbf{4 b}, \mathbf{4 d}, \mathbf{4 e}, \mathbf{4 f}, \mathbf{4 h}, \mathbf{4 i}, \mathbf{4 j}$ and $\mathbf{4 k}$ have shown good CNS depressant activity when compared with standard after $30 \mathrm{~min}$. When activity compared after $4 \mathrm{hr}$, comp $\mathbf{4 a}, \mathbf{4 b}, \mathbf{4 c}, \mathbf{4 j}$ and $\mathbf{4 k}$ have shown good CNS depressant activity when compared with standard at $30 \mathrm{mg}$ dose. Other compounds $\mathbf{4 a}, \mathbf{4 b}, \mathbf{4 g}, \mathbf{4 i}, \mathbf{4 m}$ and $\mathbf{4 n}$ have shown good CNS depressant activity at $100 \mathrm{mg}$ dose and compounds $\mathbf{4 a}, \mathbf{4 b}, \mathbf{4 c}, \mathbf{4 e}, \mathbf{4 g}, \mathbf{4 j}$ and $\mathbf{4 m}$ have shown good CNS depressant activity at $300 \mathrm{mg}$ dose. All the results are presented in Table $\mathbf{3}$ and $\mathbf{4}$. 
Table 3. Evaluation of CNS depressant activity using actophotometer after $30 \mathrm{~min}$.

\begin{tabular}{|c|c|c|c|c|c|c|}
\hline \multirow{2}{*}{ Code } & \multicolumn{3}{|c|}{ Mean change in locomotor activity Mean \pm SEM) } & \multicolumn{3}{|c|}{$\%$ inhibition in locomotor activity } \\
\hline & $\mathbf{I}$ & II & III & $\mathbf{I}$ & II & III \\
\hline $4 \mathrm{a}$ & $73 \pm 6.221^{*}$ & $59.4 \pm 13.422 * *$ & $25.6 \pm 1.965 * * *$ & 50 & 70.60 & 77.73 \\
\hline $4 \mathrm{~b}$ & $73 \pm 8.626 * *$ & $87.6 \pm 4.434 *$ & $24.4 \pm 1.939 * * *$ & 68.52 & 38.08 & 78.78 \\
\hline $4 \mathrm{c}$ & $41.2 \pm 5.490 * * *$ & $89.6 \pm 5.240^{*}$ & $98.2 \pm 2.354 \mathrm{~ns}$ & 64.17 & 22.08 & 14.60 \\
\hline $4 d$ & $78.4 \pm 12.205 * *$ & $102.8 \pm 3.397 \mathrm{~ns}$ & $23.4 \pm 0.8602 * * *$ & 31.82 & 10.60 & 79.65 \\
\hline $4 \mathrm{e}$ & $71 \pm 10.232 * * *$ & $64 \pm 4.517 * * *$ & $54.6 \pm 2.561 * * *$ & 38.78 & 44.34 & 52.52 \\
\hline $4 f$ & $72.4 \pm 8.880^{* *}$ & $65.8 \pm 5.054 * * *$ & $56.6 \pm 3.473 * * *$ & 37.04 & 42.78 & 50.78 \\
\hline $4 g$ & $103.4 \pm 2.421 \mathrm{~ns}$ & $67.8 \pm 5.791 * * *$ & $68.4 \pm 4.686^{* *}$ & 10.08 & 41.04 & 40.52 \\
\hline $4 \mathrm{~h}$ & $113.4 \pm 4.118 \mathrm{~ns}$ & $80 \pm 3.536^{* * *}$ & $60.4 \pm 5.483 * * *$ & 10.09 & 30.43 & 47.47 \\
\hline $4 \mathrm{i}$ & $97.6 \pm 7.891 \mathrm{~ns}$ & $83.8 \pm 7.723^{*}$ & $63 \pm 7.987 * * *$ & 15.13 & 27.13 & 45.21 \\
\hline $4 j$ & $102.4 \pm 8.964 \mathrm{~ns}$ & $93 \pm 4.087 \mathrm{~ns}$ & $38.8 \pm 4.883 * * *$ & 10.95 & 19.13 & 66.26 \\
\hline $4 \mathrm{k}$ & $106.8 \pm 4.883 \mathrm{~ns}$ & $109.2 \pm 6.530 \mathrm{~ns}$ & $37.2 \pm 4.224 * *$ & 7.13 & 5.04 & 67.65 \\
\hline 41 & $91.4 \pm 22.879 \mathrm{~ns}$ & $74.4 \pm 7.089 \mathrm{~ns}$ & $94.2 \pm 5.652 \mathrm{~ns}$ & 37.91 & 35.30 & 18.08 \\
\hline $4 \mathrm{~m}$ & $76.2 \pm 3.839 * * *$ & $115.8 \pm 3.967 \mathrm{~ns}$ & $90.2 \pm 4.893 *$ & 33.73 & 1.73 & 21.56 \\
\hline $4 n$ & $143.2 \pm 17.362 \mathrm{~ns}$ & $74.4 \pm 3.415^{*}$ & $37.4 \pm 2.502 * * *$ & 45.43 & 35.30 & 67.47 \\
\hline 40 & $76.8 \pm 4.341 * * *$ & $112.4 \pm 4.297 \mathrm{~ns}$ & $92 \pm 4.889^{*}$ & 33.21 & 2.26 & 20 \\
\hline $\mathrm{D}$ & \multicolumn{3}{|c|}{$30.2 \pm 2.922$} & \multicolumn{3}{|c|}{47} \\
\hline $\mathrm{C}$ & \multicolumn{6}{|c|}{$115 \pm 7.791$} \\
\hline
\end{tabular}

Each value represents the mean_SEM significantly different from the control at $P<0.05$; ns denotes not significant at $P<0.05$ (Student's $t$-test); $* P<0.05, * * P<0.01, * * * P>0.05$ locomotor activity score was measured for $10 \mathrm{~min} . \mathrm{D}=\mathrm{Diazepam} ; \mathrm{C}=\mathrm{control} ; \mathrm{I}=30 \mathrm{mg} / \mathrm{kg}, \mathrm{II}=100 \mathrm{mg} / \mathrm{kg}, \mathrm{III}=300$ $\mathrm{mg} / \mathrm{kg}$.

Table 4. Evaluation of CNS depressant activity using actophotometer after 4 h.

\begin{tabular}{|c|c|c|c|c|c|c|}
\hline \multirow{2}{*}{ Code } & \multicolumn{3}{|c|}{ Mean change in locomotor activity (Mean \pm SEM) } & \multicolumn{3}{|c|}{$\%$ inhibition in locomotor activity } \\
\hline & $\mathbf{I}$ & II & III & $\mathbf{I}$ & II & III \\
\hline $4 \mathrm{a}$ & $40 \pm 9.965 * * *$ & $33.8 \pm 1.655 * * *$ & $26 \pm 2.168 * * *$ & 46.14 & 54.50 & 44.21 \\
\hline $4 \mathrm{~b}$ & $75.2 \pm 11.608 *$ & $46.8 \pm 5.324 * * *$ & $54.8 \pm 10.017 * * *$ & 39.54 & 62.37 & 55.94 \\
\hline $4 \mathrm{c}$ & $36.8 \pm 6.094 * * *$ & $58.2 \pm 5.161 * * *$ & $64 \pm 4.231 * * *$ & 39.53 & 43.56 & 42.28 \\
\hline $4 d$ & $84 \pm 7.817^{*}$ & $138.2 \pm 4.432 \mathrm{~ns}$ & $19 \pm 1.414 * * *$ & 32.47 & 11.09 & 43.24 \\
\hline $4 \mathrm{e}$ & $133 \pm 13.657 \mathrm{~ns}$ & $98 \pm 3.347 \mathrm{~ns}$ & $51.8 \pm 12.212 * * *$ & 6.91 & 21.22 & 58.36 \\
\hline $4 f$ & $116.8 \pm 6.248 \mathrm{~ns}$ & $82.4 \pm 5.115 \mathrm{~ns}$ & $120.2 \pm 10.288 * *$ & 9.32 & 33.76 & 3.49 \\
\hline $4 \mathrm{~g}$ & $99.2 \pm 3.839 *$ & $77.4 \pm 5.662 * * *$ & $63.4 \pm 5.605 * * *$ & 20.25 & 37.78 & 49.03 \\
\hline $4 \mathrm{~h}$ & $114.2 \pm 6.053 \mathrm{~ns}$ & $99.8 \pm 2.922 *$ & $71.8 \pm 3.089 * * *$ & 8.19 & 19.77 & 42.28 \\
\hline $4 \mathrm{i}$ & $93 \pm 9.354 \mathrm{~ns}$ & $78.8 \pm 9.452 *$ & $74.6 \pm 11.365^{* *}$ & 25.24 & 36.65 & 40.03 \\
\hline $4 \mathrm{j}$ & $73.4 \pm 2.619 * * *$ & $93.4 \pm 5.335^{*}$ & $42 \pm 8.620 * * *$ & 40.99 & 24.91 & 66.23 \\
\hline $4 \mathrm{k}$ & $45 \pm 8.444 * * *$ & $93.4 \pm 9.903 *$ & $28 \pm 2.713 * * *$ & 43.56 & 24.91 & 38.90 \\
\hline 41 & $182 \pm 9.706 \mathrm{~ns}$ & $128.2 \pm 2.375 \mathrm{~ns}$ & $134.8 \pm 9.431 \mathrm{~ns}$ & 4.6 & 3.53 & 8.36 \\
\hline $4 m$ & $87.8 \pm 10.758^{*}$ & $73.6 \pm 7.891 * *$ & $52.4 \pm 8.035^{* * *}$ & 29.42 & 40.83 & 57.87 \\
\hline $4 n$ & $42.6 \pm 1.364 * * *$ & $34.2 \pm 1.020 * * *$ & $24.6 \pm 2.561 * * *$ & 45.17 & 41.63 & 43.89 \\
\hline 40 & $97.6 \pm 6.645 \mathrm{~ns}$ & $80.2 \pm 5.229 * *$ & $118.8 \pm 11.795 \mathrm{~ns}$ & 35.53 & 21.54 & 4.50 \\
\hline $\mathrm{D}$ & \multicolumn{3}{|c|}{$75 \pm 11.150$} & \multicolumn{3}{|c|}{47.42} \\
\hline $\mathrm{C}$ & \multicolumn{6}{|c|}{$124.4 \pm 5.758$} \\
\hline
\end{tabular}

Each value represents the mean_SEM significantly different from the control at $P<0.05$; ns denotes not significant at $P<0.05$ (Student's $t$-test); $* P<0.05, * * P<0.01, * * * P>0.05$ locomotor activity score was measured for $10 \mathrm{~min} . \mathrm{D}=\mathrm{Diazepam} ; \mathrm{C}=\mathrm{control} ; \mathrm{I}=30 \mathrm{mg} / \mathrm{kg}, \mathrm{II}=100 \mathrm{mg} / \mathrm{kg}, \mathrm{III}=300$ $\mathrm{mg} / \mathrm{kg}$.

\section{Anticonvulsant Activity}

All the tested compounds showed protection against MES test (Table 5) indicative of their ability to inhibit the seizure spread. Compounds $\mathbf{4 g}$ showed protection against the MES model at $30 \mathrm{mg} / \mathrm{kg}$ and compounds $4 \mathbf{a}, \mathbf{4 e}, \mathbf{4 f}$ showed protection at dose level of $100 \mathrm{mg} / \mathrm{kg}$, while compounds $\mathbf{4 b}, \mathbf{4 c}, \mathbf{4 h}, \mathbf{4 i}, \mathbf{4 n}$ showed protection at dose level of $300 \mathrm{mg} / \mathrm{kg}$ after $0.5 \mathrm{~h}$. Compounds $\mathbf{4 d}$ and $\mathbf{4 l}$ showed protection against the MES model at $30 \mathrm{mg} / \mathrm{kg}$ and compounds $\mathbf{4 f}$, $\mathbf{4 m}$ showed protection at dose level of $100 \mathrm{mg} / \mathrm{kg}$ while compounds $\mathbf{4 a}, \mathbf{4 b}, \mathbf{4 c}, \mathbf{4 e}, \mathbf{4 g}, \mathbf{4 h}, \mathbf{4 i}, \mathbf{4 j}, \mathbf{4 k}$ and $\mathbf{4 n}$ showed 
protection at dose level of $300 \mathrm{mg} / \mathrm{kg}$ at $4 \mathrm{~h}$. Compound $\mathbf{4 m}$ was found to be more effective but short acting as $4 \mathrm{~h}$ protection requires the dose of $100 \mathrm{mg} / \mathrm{kg}$. The compounds $4 \mathbf{f}$ showed activity both at $0.5 \mathrm{~h}$ and $4 \mathrm{~h}$ period at dose level of $100 \mathrm{mg} / \mathrm{kg}$ indicating that compounds are effective and long acting. The compounds $\mathbf{4 b}, \mathbf{4 c}, \mathbf{4 f}, \mathbf{4 i}$ and $\mathbf{4 n}$ showed activity both at $0.5 \mathrm{~h}$ and $4 \mathrm{~h}$ period at dose level of $300 \mathrm{mg} / \mathrm{kg}$ indicating that compounds are less potent and long acting. The compounds $\mathbf{4 d}, \mathbf{4 l}$ showed activity at dose level of $30 \mathrm{mg} / \mathrm{kg}$ and $\mathbf{4 k}$ and $\mathbf{4 m}$ showed activity at dose level of $100 \mathrm{mg} / \mathrm{kg}$ only at $4 \mathrm{~h}$ indicating that compounds have sustained release activity.

Most of the compounds were found to be active in the sc PTZ test (Table 5) a test used to identify compounds that elevate seizure threshold. The Compounds $\mathbf{4 a}, \mathbf{4 b}$ and $\mathbf{4 k}$ showed activity at a dose of $100 \mathrm{mg} / \mathrm{kg}$ while, compounds $\mathbf{4 g}$, $\mathbf{4 h}, \mathbf{4 i}$ and $\mathbf{4 n}$ showed activity at dose of $300 \mathrm{mg} / \mathrm{kg}$ at $0.5 \mathrm{~h}$. The Compounds $4 \mathbf{a}, \mathbf{4 b}, \mathbf{4 h}, \mathbf{4 i}, \mathbf{4 k}$ and $\mathbf{4 n}$ have shown activity at dose of $300 \mathrm{mg} / \mathrm{kg}$ at $4 \mathrm{~h}$. No compounds were found active at dose level of $30 \mathrm{mg} / \mathrm{kg}$. The compounds $\mathbf{4 h}$, $\mathbf{4 i}$ and $4 \mathrm{n}$ have shown activity at $300 \mathrm{mg} / \mathrm{kg}$ at $0.5 \mathrm{~h}$ and $4 \mathrm{~h}$ indicating that these compounds have moderate activity. For several classes of CVS active substances, Hansch and Leo found that BBB penetration is optimal when the log $P$ values are in the range of 1.5-2.7, with 2.1 mean value [32, 33]. In this study, we attempted to correlate the anticonvulsant activity of synthesized compounds with $\log P$ value. The compounds $4 \mathbf{c}, \mathbf{4 d}, \mathbf{4 e}, \mathbf{4 f}, \mathbf{4 j}, \mathbf{4 l}, \mathbf{4 m}$ and $\mathbf{4 n}$ showed absence of activity at all dose level. These compounds were having log P more than 5 while, compounds $\mathbf{4 g}$ found to be active with short duration of action.

Table 5. Anticonvulsant screening of compounds.

\begin{tabular}{|c|c|c|c|c|}
\hline \multirow{2}{*}{ Compounds } & \multicolumn{2}{|c|}{ MES screen } & \multicolumn{2}{|c|}{ Sc PTZ } \\
\hline & $0.5 \mathrm{~h}$ & $4 \mathrm{~h}$ & $0.5 \mathrm{~h}$ & $4 \mathrm{~h}$ \\
\hline $4 \mathrm{a}$ & 100 & 300 & 100 & 300 \\
\hline $4 \mathrm{~b}$ & 300 & 300 & 100 & 300 \\
\hline $4 \mathrm{c}$ & 300 & 300 & --- & --- \\
\hline $4 d$ & ---- & 30 & --- & --- \\
\hline $4 \mathrm{e}$ & 100 & 300 & --- & --- \\
\hline $4 \mathrm{f}$ & 100 & 100 & --- & --- \\
\hline $4 \mathrm{~g}$ & 30 & 300 & 300 & \\
\hline $4 \mathrm{~h}$ & 300 & 300 & 300 & 300 \\
\hline $4 \mathrm{i}$ & 300 & 300 & 300 & 300 \\
\hline $4 \mathrm{j}$ & --- & 300 & --- & --- \\
\hline $4 \mathrm{k}$ & --- & 100 & 100 & 300 \\
\hline 41 & -- & 30 & --- & --- \\
\hline $4 \mathrm{~m}$ & --- & 100 & --- & --- \\
\hline $4 n$ & 300 & 300 & 300 & 300 \\
\hline 40 & -- & 300 & --- & --- \\
\hline Phenytoin & 30 & 30 & $\mathrm{x}$ & $\mathrm{x}$ \\
\hline
\end{tabular}

a Doses of 30,100 and $300 \mathrm{mg} / \mathrm{kg}$ of the compound were administered and the protection measured after 0.5 and $4 \mathrm{~h}$. The figures indicate the minimal dose required to cause protection in $50 \%$ or more of the animals. The dash (---) indicates the absence of anticonvulsant activity. $\mathrm{x}$ denotes not tested.

\section{COMPUTATIONAL PARAMETERS}

\section{The Docking Study}

Docking study of synthesized compounds 4(a-o) was performed using VLife MDS 4.3 package for anticonvulsant activity [34]. With this purpose, crystal structure of the biological target GABA-A receptor (PDB ID: 4COF) [35] was obtained from the Protein Data Bank in order to prepare protein for docking study. Docking procedure was followed using the standard protocol implemented in VLife MDS 4.3 package and synthesized compounds $\mathbf{4 ( a - o )}$ were docked against three dimensional structure of GABA-A receptor.

Benzothiazepines is an example of bioisosteric replacement of benzodiazepines, enhance the effect of the neurotransmitter gamma aminobutyric acid (GABA) at the GABAA receptor, acts as a positive allosteric modulator by increasing the total conduction of chloride ions across the neuronal cell membrane [36], resulting in sedative, hypnotic (sleep-inducing), anticonvulsant and muscle relaxant properties [37]. Thus, synthesizing compounds have been docked against GABA-A receptor to understand the binding interactions and the data obtained would be very helpful to achieve further modification of lead for greater inhibitory activity. The docking study revealed 
that the synthesized compounds were held in active pocket by forming various hydrophobic and van der Waal's interaction with GABA-A receptor. The various hydrophobic interactions formed by synthesized compounds with amino acid residues like THR96, TYR97, PHE98, ASP101, SER104, PHE105, VAL106, LYS112, LEU128, ILE130, and THR132. The compounds have formed various van der Waal's interactions with amino acid residues like PHE63, GLN65, VAL93, PRO94, THR96, TYR97, PHE98, ASP101, SER104, PHE105, VAL106, HIS107, GLY108, LYS112, ARG114, TYR126, LEU128, ILE130, and THR132. The binding interactions of most active compounds (4a, 4b, 4h, 4i and 4n) with the GABA-A receptor are shown in Fig. (3).
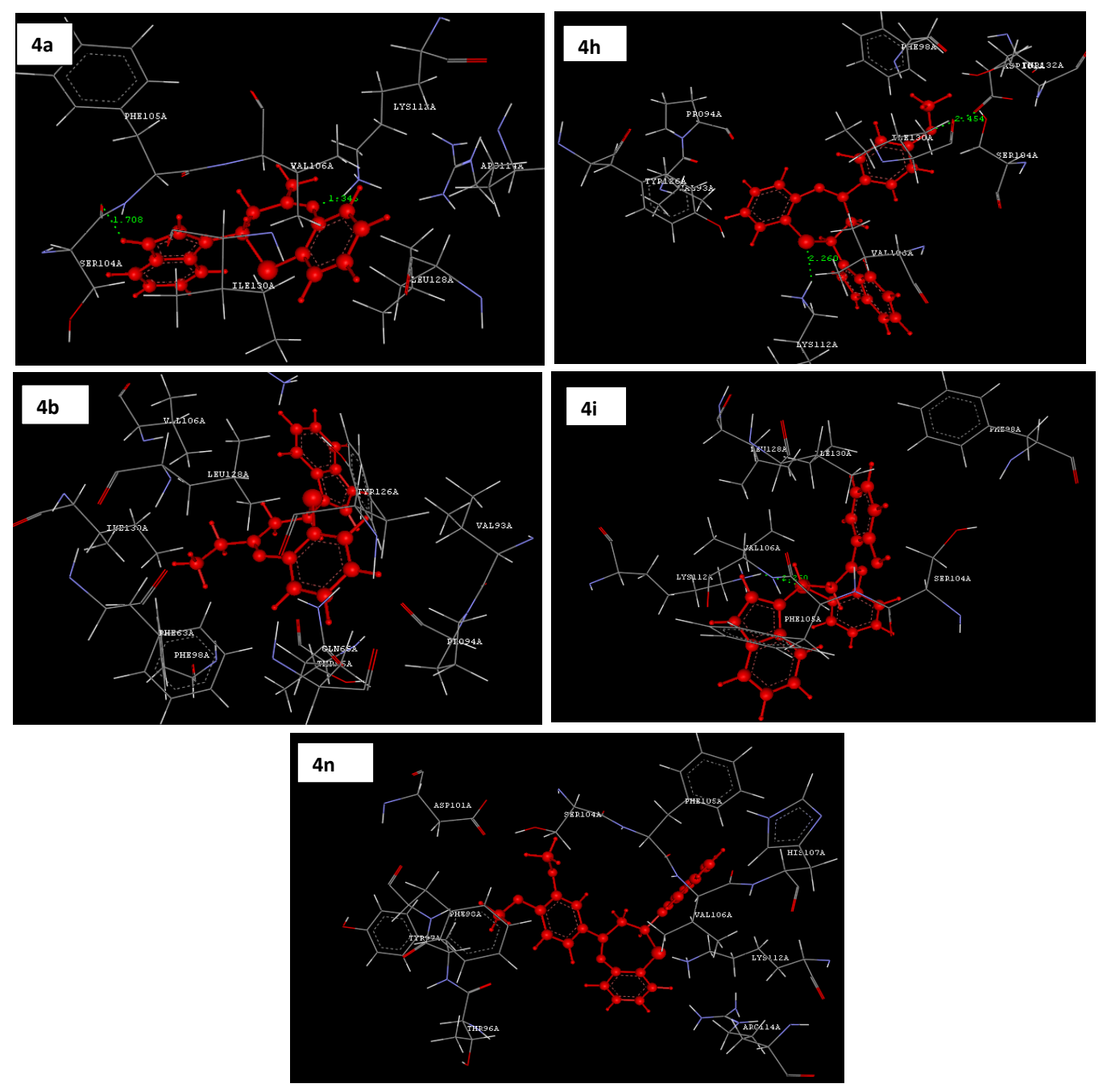

Fig. (3). Docking images of compounds $4 \mathbf{a}, \mathbf{4 b}, \mathbf{4 h}, \mathbf{4 i}$ and $\mathbf{4 n}$ with GABA-A receptor (PDB ID: 4COF). Ligands are shown as ball and stick with red color.

The compound 4a had shown good binding interactions with GABA-A receptor and benzothiazepine and indole nucleus were held in active pocket by forming various interactions with amino acids like SER104, PHE105, VAL106, LYS112, LEU128 and ILE130. The both nitrogen atoms i.e. nitrogen of benzothiazepine and indole showed hydrogen bonding with amino acids LYS112 (1.34 $\AA$ ) and SER104 (1.70 $\AA)$, respectively. The $-\mathrm{CH}_{3}$ group was also held into the active pocket by forming the hydrophobic interaction with amino acid LYS112. The compound $\mathbf{4 b}$ was held in active pocket by forming various hydrophobic and van der Waal's interactions with amino acids like PHE63, GLN65, VAL93, PRO94, THR96, PHE98, VAL106, LYS112, TYR126, LEU128, and ILE130. The -C2H5 group was held in active pocket by forming interactions with amino acid ILE130. The compound had not shown any hydrogen bonding with GABA-A receptor and this may be the reason for less activity of compound $\mathbf{4 b}$ than compound $\mathbf{4 a}$.

The compounds $\mathbf{4 h}, \mathbf{4 i}$ and $\mathbf{4 n}$ were equipotent showing the protection in both the model at the dose of $300 \mathrm{mg} / \mathrm{kg}$. The docking of compound $\mathbf{4 h}$ with GABA-A receptor showed good binding interactions with amino acid residues like 


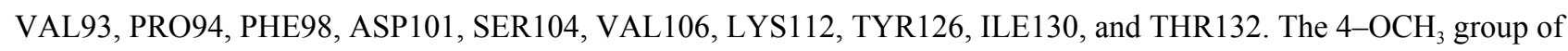
phenyl was held deep into the cavity by forming hydrophobic interactions with amino acids like PHE98, ASP101, SER104, ILE130 and THR132. The sulphur of benzothiazepine ring and oxygen of $-\mathrm{OCH}_{3}$ had formed hydrogen bonding with amino acids LYS112 (2.26 $)$ ) and SER104 (2.45 $\AA$ ), respectively. The compound $4 \mathbf{i}$ was held into active pocket by forming various hydrophobic and van der Waal's interactions with amino acid residues like PHE98, SER104, PHE105, VAL106, LYS112, LEU128, and ILE130. The sulphur atom of benzothiazepine ring had shown hydrogen bonding with amino acid LYS112 (2.26 ̊). The compound 4n has shown various hydrophobic and van der Waal's interactions with amino acids residues like THR96, TYR97, PHE98, ASP101, SER104, PHE105, VAL106, HIS107, LYS112, and ARG114. The compound 4n had not shown any hydrogen bonding with GABA-A receptor. Both the $-\mathrm{OCH}_{3}$ group of phenyl ring held in active pocket by forming hydrophobic interactions with amino acids TYR97, PHE98 and SER104. Thus, the results obtained (activity data and docking results) support that the compounds $\mathbf{4 a}, \mathbf{4 b}$, 4h, $\mathbf{4 i}$ and $\mathbf{4 n}$ might exert their anticonvulsant action as GABA-A agonist.

\section{EXPERIMENTAL}

\section{Chemistry}

All reagents and solvents were used as obtained from the supplier or recrystallized/redistilled unless otherwise noted. Most chemicals purchased were of Merck grade. All chemicals were crystallized or redistilled before use. Silica gel $G$ was used for thin layer chromatography. Melting points were taken in open capillaries using melting point apparatus and are uncorrected. ${ }^{1} \mathrm{H}$ NMR spectra were obtained using a BRUKER AVANCE II 400 spectrometer. Tetramethylsilane was used as an internal standard. Mass spectra were obtained using Time of Flight. The IR spectra were obtained using a JASCO FTIR 4000 spectrometer. Elemental analyses were carried out on FLASHEA 1112 series and observed values were within $\pm 0.4 \%$ of theoretical values for $\mathrm{C}, \mathrm{H}$ and $\mathrm{N}$. The synthetic microwave oven used was of Scientific Catalyst synthesis (Cata R).

Indole-3-carbaldehyde 2 was obtained as per the given procedure [38]. The product was recrystallized from ethanol to give $9.7 \mathrm{~g}$ of pale straw crystals. IR $(\mathrm{KBr}): v=3448 \mathrm{~cm}^{-1}(\mathrm{NH}), 1720(\mathrm{C}=\mathrm{O}) .-{ }^{1} \mathrm{H}-\mathrm{NMR}\left(\mathrm{CDCl}_{3}\right): \delta=7.2-8.1(\mathrm{~m}$, $4 \mathrm{H}$, aromatic protons), 8.2 (s, 1H, $\mathrm{C}_{2} \mathrm{H}$ - indole), 9.7 (s, 1H, CHO), 10.8 (s, 1H, NH- indole). MS m/z: 145.

\section{General Procedure for the Synthesis of Chalcones 3(a-o).}

Chalcone derivatives were synthesized by condensing indole-3-carbaldehyde with various aromatic ketones according to the Claisen-Schmidt condensation [39] reported in the literature [40, 41]

\section{General Procedure for the Synthesis of Indolyl Substituted Benzothiazepine4a -o}

In a borosil beaker, (1.85 g, $0.01 \mathrm{~mol})$ 1-methyl-3-(1H-indol-3-yl)-2-propen-1-one, (1.25 g, $0.01 \mathrm{~mol}) 2$ aminothiophenol and a pinch of zirconium oxy chloride catalyst was taken and mixed uniformly. This reaction mixture was irradiated under microwave for $3 \mathrm{~min}$. After the completion of reaction, the reaction mixture was allowed to attain room temperature and treated with cold water to remove the catalyst. The solid separated was filtered off, washed with water and recrystallized from ethanol to give $75 \%$ of $4 \mathrm{a}, \mathrm{mp} 80^{\circ} \mathrm{C}$.

\section{Antihypertensive Activity}

Adult Sprague- Dawley rats of either sex weighing 200-250 g were used. Animals were housed 4-5 per cage and exposed to a $12 \mathrm{~h}$ light-dark cycle. All rats were allowed food and water. Six rats were used for each dose and compound. The control group received $0.2 \% \mathrm{CMC}(0.5 \mathrm{ml} / 100 \mathrm{~g}$ i.p. $)$ only. To reduce spontaneous variation in blood pressure animals were adjusted to the experimental cage 3-4 times before the start of the experiment for a period of 30-60 min. changes of blood pressure were measured by using a tail- cuff method. Automatic measurement of systolic blood pressure was provided by a pressure transducer (ML 125/R pulse detector) of Power Lab Data Acquisition System. All test compounds were suspended in $0.2 \% \mathrm{CMC}$ and administered intraperitoneally $(5 \mathrm{mg} / \mathrm{kg}) \mathrm{in} \mathrm{rats.}$

\section{Behavioural Activity}

The activity was measured as digital score using actophotometer with the ip administration of $\mathrm{drug}(30 \mathrm{mg} / \mathrm{kg})$ to mice. The mice were placed in the box and the behavior was noted for $10 \mathrm{~min}$. Further, the animals were treated with the drug and after $0.5 \mathrm{~h}$ and $4 \mathrm{~h}$ drug administration the animal were re-tested. The activity score was noted and based 
on these results, \% decrease in locomotor activity was calculated. Diazepam was used as a standard drug.

\section{Anticonvulsant Activity}

Male Swiss albino mice (CF-1 strain, 20-30 g) were used as experimental animals. All the test compounds were suspended in $0.5 \%$ methyl cellulose in the case of MES and sc PTZ induced seizure models and $30 \%$ PEG 200 for behavioral activity at our lab. The animals were maintained at an ambient temperature of $25 \pm 2^{\circ} \mathrm{C}$, in groups of five per cage under standard laboratory conditions, receiving standard laboratory chow and water ad libitum. A $12 \mathrm{~h}: 12 \mathrm{~h}$ light/dark cycle was maintained throughout the experimental studies. All the tests have been performed in accordance with the guidelines laid out by the Institutional Animal Ethics Committee. (CPCSEA Reg. No. CPCSEA/IAEC/Pharm.Chem.06/2011-12).

All the test compounds were administered intraperitoneally in a volume of $0.01 \mathrm{~mL} / \mathrm{g}$ for mice at doses of 30,100 and $300 \mathrm{mg} / \mathrm{kg}$. Anticonvulsant activity was assessed after $30 \mathrm{~min}$ and $4 \mathrm{~h}$ of drug administration. The preliminary anticonvulsants (MES and sc PTZ) evaluations were done using reported procedures using phenytoin as a standard drug.

\section{CONCLUSION}

From the research work under taken and the results obtained it can be concluded that 2-(1H-indol-3-yl)-4-(alkyl/ substituted phenyl)-2, 3-dihydro-1, 5-benzothiazepine derivatives were obtained in solvent-free condition under microwave-irradiation in good yield and in shorter reacton times. These hybrid derivatives possess significant antihypertensive activity and have very good potential for the development as novel antihypertensive agents. The compounds have also shown good CNS depressant activity and anticonvulsant activity, when compared with the standard drug diazepam. Most of the compounds were found to be active in the sc PTZ test. These hybrid heterocyclic compounds thus have shown dual antihypertensive and CNS depressant, anticonvulsant activity and are biologically potential molecules. Molecular docking studies revealed that the investigated compounds had shown good binding interactions with GABA-A receptor and might exert their anticonvulsant activity as GABA-A receptor agonist.

\section{LIST OF ABBREVIATIONS}

$\begin{array}{lll}\text { MS } & = & \text { Mass Spectroscopy } \\ \text { MES } & = & \text { Maximal Electroshock Seizure } \\ \text { NMR } & = & \text { Nuclear Magnetic Resonance } \\ \text { Sc PTZ } & = & \text { Subcutaneous pentylenetetrazole }\end{array}$

\section{SUPPLEMENTARY DATA}

Supplementary data $\left({ }^{1} \mathrm{HNMR},{ }^{13} \mathrm{CNMR}\right.$, mass, IR spectras of representative compounds) associated with this article can be found.

\section{CONFLICT OF INTEREST}

The authors confirm that this article content has no conflict of interest.

\section{ACKNOWLEDGEMENTS}

We as the authors are thankful to the Chairman, Maulana Azad Education Trust and Principal Y. B. Chavan College of Pharmacy, Aurangabad, for providing laboratory facility and authors express their gratitude towards SAIF, Panjab University, and Chandigarh for providing Mass and NMR Spectral Analysis and University of Pune for providing elemental analysis of the synthesized compounds.

\section{REFERENCES}

[1] Goodman LS, Hardman JG, Limbard LE, Gilman AG, Eds. Goodman and Gilman's the Pharmacological Basis of Therapeutics. 10 $0^{\text {th }}$ ed. New York: Mc Graw Hill C2001.

[2] Joshi G, Burnett J, Abraham D. Cardiac drugs: Antianginal, Vasodilators and antiarrhythmic. In: Abraham D, Ed. Burger's Medicinal Chemistry and Drug Discovery, Cardiovascular agents and endocrines. USA: Wiley Science Publications 2003. [http://dx.doi.org/10.1002/0471266949.bmc041]

[3] Belusa J, Hruskova V, Haas Z, Kaminska Z, Picha F, Dusek J. CS 274, 213, Chem Abstr 1992; 118: 2459706. 
[4] Vaghy L, Williams S, Schwartz AA. Symposium: Hypertension-The Heart and Kidney. Am J Cardiol 1987; 60(17): 1-132. [PMID: 3604922]

[5] Bacon KB, Westwick J, Camp RD. Potent and specific inhibition of IL-8-, IL-1 $\alpha$ - and IL-1 $\beta$-induced in vitro human lymphocyte migration by calcium channel antagonists. Biochem Biophys Res Commun 1989; 165(1): 349-54. [http://dx.doi.org/10.1016/0006-291X(89)91076-0] [PMID: 2686646]

[6] Hirano K, Kanaide H, Abe S, Nakamura M. Effects of diltiazem on calcium concentrations in the cytosol and on force of contractions in porcine coronary arterial strips. Br J Pharmacol 1990; 101(2): 273-80. [http://dx.doi.org/10.1111/j.1476-5381.1990.tb12700.x] [PMID: 2257435]

[7] Aoki K, Sato K, Kondo S, Yamamoto M. Hypotensive effects of diltiazem to normals and essential hypertensives. Eur J Clin Pharmacol 1983; 25(4): 475-80.

[http://dx.doi.org/10.1007/BF00542114] [PMID: 6360693]

[8] Burris JF, Weir MR, Oparil S, Weber M, Cady WJ, Stewart WH. An assessment of diltiazem and hydrochlorothiazide in hypertension. Application of factorial trial design to a multicenter clinical trial of combination therapy. JAMA 1990; 263(11): 1507-12. [http://dx.doi.org/10.1001/jama.1990.03440110073031] [PMID: 2407872]

[9] Weiss K, Fitscha P, Gazsó A, Gludovacz D, Sinzinger H. Calcium blockers: antiatherogenic action by influencing the prostaglandin-system. Prog Clin Biol Res 1989; 301: 353-7.

[PMID: 2529552]

[10] D’Alonzo AJ, Hess TA, Darbenzio RB, Sewter JC, Raymond R. Effects of intracoronary cromakalim, pinacidil, or diltiazem on cesium chloride-induced arrhythmias in anesthetized dogs under conditions of controlled coronary blood flow. J Cardiovasc Pharmacol 1993; 21(4): 677-83.

[http://dx.doi.org/10.1097/00005344-199304000-00024] [PMID: 7681916]

[11] Kuzelova M, Svec P. The effect of calcium channel blockers in experimental myocardial infarct in rats. Cesk Farm 1993; $42(3)$ : 124-6. [PMID: 8402964]

[12] Wang L, Zhang P, Zhang X, Zhang Y, Li Y, Wang Y. Synthesis and biological evaluation of a novel series of 1,5-benzothiazepine derivatives as potential antimicrobial agents. Eur J Med Chem 2009; 44(7): 2815-21. [http://dx.doi.org/10.1016/j.ejmech.2008.12.021] [PMID: 19144450]

[13] Nikalje A. Synthesis of 1,5-benzothiazepins as a potential CNS and CVS agents. Int J Chem Sci 2007; 5(1): $290-6$.

[14] Kaur H, Kumar S, Chaudhary A, Kumar A. Synthesis and biological evaluation of some new substituted benzoxazepine and benzothiazepine as antipsychotic as well as anticonvulsant agents. Arab J Chem 2012; 5: 271-83. [http://dx.doi.org/10.1016/j.arabjc.2010.09.011]

[15] Ahuja P, Siddiqui N. Anticonvulsant evaluation of clubbed indole-1,2,4-triazine derivatives: a synthetic approach. Eur J Med Chem 2014; 80: 509-22.

[http://dx.doi.org/10.1016/j.ejmech.2014.04.043] [PMID: 24813879]

[16] Falcó JL, Piqué M, González M, et al. Synthesis, pharmacology and molecular modeling of N-substituted 2-phenyl-indoles and benzimidazoles as potent GABA(A) agonists. Eur J Med Chem 2006; 41(8): 985-90.

[http://dx.doi.org/10.1016/j.ejmech.2006.03.031] [PMID: 16764969]

[17] Stanton JL, Ackerman MH. Synthesis and anticonvulsant activity of some tetracyclic indole derivatives. J Med Chem 1983; 26(7): 986-9. [http://dx.doi.org/10.1021/jm00361a010] [PMID: 6864737]

[18] Kamiński K, Obniska J. Design, synthesis, and anticonvulsant activity of $N$-phenylamino derivatives of 3,3-dialkyl-pyrrolidine-2,5-diones and hexahydro-isoindole-1,3-diones. Bioorg Med Chem 2008; 16(9): 4921-31. [http://dx.doi.org/10.1016/j.bmc.2008.03.037] [PMID: 18375131]

[19] Rani P, Bajaj K, Srivastava V, Kumar A. Synthesis and anti-convulsant activity of heterocyclic indole derivatives. Arzneim Forsch Drug Res 2003; 53: 301 .

[20] Patil P, Bari S. Synthesis and Antidepressant Activity of Some New 5-(1H-Indol-3-yl)-3-(substituted aryl)-4, 5-dihydroisoxazoline Derivatives. J Chem 2013; 7: 1-7.

[21] Panda SS, Chowdary PV. Synthesis of novel indolyl-pyrimidine antiinflammatory, antioxidant and antibacterial agents. Indian J Pharm Sci 2008; 70(2): 208-15.

[http://dx.doi.org/10.4103/0250-474X.41457] [PMID: 20046714]

[22] Sharma S, Srivastava V, Kumar A. Synthesis of some newer indolyl-thiadiazolyl-pyrazolines as potential anti-inflammatory agent. Indian J Chem 2002; 41B: 2647-54.

[23] Bajaj K, Srivastava V, Lata S, Chandra R, Kumar A. Synthesis of new benzothia/oxazepinyl indoles as an antipsychotic agents. Indian J Chem 2003; 42B: 1723-8.

[24] Nikalje A, Shaikh S, Mulay A, Khan F, Sangshetti J. Molecular sieves promoted, ultrasound-mediated synthesis, biological evaluation and docking study of 3-(5-substituted-1, 3, 4-thiadiazol-2-ylimino) indolin-2-ones as anticonvulsant agents. Arch Pharm Chem Life Sci 2014; 347: $1-12$.

[25] Nikalje A, Vyawahare D, Ghodke M. Green synthesis and pharmacological screening of novel 1, 5-benzothiazepines as CNS agents. Int J 
Pharm Pharma Sci 2010; 2(2): 27-9.

[26] Pant S, Singhal B, Upreti M, Pant U. Syntheses of 1,5-Benzothiazepines. Part 20. Syntheses of 8-Substituted-2,5-dihydro-2-(4-Ndimethylaminophenyl)-4-(4-methoxyphenyl)-1,5-benzothiazepines. Molecules 1998; 3: 159-63. [http://dx.doi.org/10.3390/30600159]

[27] Bariwal JB, Upadhyay KD, Manvar AT, et al. 1,5-Benzothiazepine, a versatile pharmacophore: a review. Eur J Med Chem 2008; 43(11): 2279-90. [http://dx.doi.org/10.1016/j.ejmech.2008.05.035] [PMID: 18639369]

[28] Patel V, Desai K. Microwave assisted heterocyclization: A rapid and efficient synthesis of 1,5-benzothiazepines. Indian J Chem 2004; 43(1): 199-201.

[29] Nagawade R, Shinde D. Zirconium (IV) Chloride - Catalysed Reaction of Indoles: An Expeditious Synthesis of Bis(indolyl)methanes. Bull Korean Chem Soc 2005; 26(12): 1962-4 [http://dx.doi.org/10.5012/bkcs.2005.26.12.1962]

[30] Shafiee A, Rastkary N, Jorjani M, Shafaghi B. Synthesis and antihypertensive activities of new 1,4-dihydropyridine containing nitroimidazolyl substituent with a nitrooxy group at the 3-ester position. Arch Pharm Med Chem 2002; 2: 69-76. [http://dx.doi.org/10.1002/1521-4184(200203)335:2/3<69::AID-ARDP69>3.0.CO;2-8]

[31] Boissier J, Simon P. Behavioral despair in mice: a primary screening test for antidepressants. Arch Int Pharmacodyn Ther 1965; 158: 212-4. [PMID: 5854047]

[32] Hansch C, Leo A. Substituent Constant for Correlation Analysis in Chemistry and Biology. New York: Wiley 1979.

[33] Yang YJ, Zhao JH, Pan XD, Zhang PC. Synthesis and antiviral activity of phthiobuzone analogues. Chem Pharm Bull (Tokyo) 2010; 58(2): 208-11. [http://dx.doi.org/10.1248/cpb.58.208] [PMID: 20118580]

[34] Life Molecular Design Suite 4.3, VLife Sciences Technologies Pvt. Ltd. Available at: www.Vlifesciences.com.

[35] Miller PS, Aricescu AR. Crystal structure of a human GABA receptor. Nature 2014; 512(7514): $270-5$. [http://dx.doi.org/10.1038/nature13293] [PMID: 24909990]

[36] Bertilsson L. Mechanism of action of benzodiazepines - the GABA hypothesis. Acta Psychiatr Scand Suppl 1978; 274(274): 19-26. [http://dx.doi.org/10.1111/j.1600-0447.1978.tb02383.x] [PMID: 216234]

[37] Kumar A. Anticonvulsant and muscle relaxant properties. Am J Pharm Sci 2013; 1(6): 116-20.

[38] Rabjohn N. Organic Synthesis Collective. New York: John Wiley and Sons 2005; pp. 539-41.

[39] Furniss B, Hannaford A, Smith P, Tatchell A. Vogel's Textbook of Practical Organic Chemistry. 2005.

[40] Rajendraprasad Y, Ranikumar P, Srinivasrao A, Rambabu R. Synthesis of some 4'-aminochalcones and their antiinflammatory and antimicrobial activity. Asian J Chem 2009; 21(2): 907-14.

[41] Jhata Y, Verma B. Synthesis and antimicrobial activity of 1, 3, 4-oxadiazole. Ind J Het Chem 2005; 14(4): 357-8.

(C) Nikalje et al.; Licensee Bentham Open.

This is an open access article licensed under the terms of the Creative Commons Attribution-Non-Commercial 4.0 International Public License (CC BY-NC 4.0) (https://creativecommons.org/licenses/by-nc/4.0/legalcode), which permits unrestricted, non-commercial use, distribution and reproduction in any medium, provided the work is properly cited. 